\title{
INTRINSIC CALCIUM DYNAMICS CONTROL BOTULINUM TOXIN A SUSCEPTIBILITY IN DISTINCT NEURONAL POPULATIONS
}

\author{
Carlotta Grumelli ${ }^{1}$, Irene Corradini ${ }^{1}$, Michela Matteoli ${ }^{1,2}$ and Claudia Verderio ${ }^{1}$ \\ ${ }^{1}$ CNR Institute of Neuroscience and Dept. of Medical Pharmacology, University of Milan, Via Vanvitelli 32, 20129 \\ Milano. ${ }^{2}$ IRCCS Fondazione Don Gnocchi, Milano, Italy
}

Address correspondence to Claudia Verderio or Michela Matteoli, Dept. of Medical Pharmacology University of Milano, Via Vanvitelli 32, 20129 Milano ITALY, Tel. 39-02-50317097; Fax: 0039-02-7490574; E-mail: c.verderio@in.cnr.it; m.matteoli@in.cnr.it; 


\section{ABSTRACT}

SNAP-25 is a SNARE protein implicated in exocytosis and in the negative modulation of voltage gated calcium channels. We have previously shown that GABAergic synapses, which express SNAP-25 at much lower levels relative to glutamatergic ones, are characterized by a higher calcium responsiveness to depolarization and are largely resistant to botulinum toxin A. We show here that silencing of SNAP-25 in glutamatergic neurons, a procedure which increases KCl-induced calcium elevations, confers these synapses with toxin resistance. Since it is known that calcium reverts the efficacy of botulinum A, we investigated whether the lower effectiveness of the toxin in inhibiting GABAergic vesicle cycling might be attributable to higher evoked calcium transients of inhibitory neurons. We demonstrate that either expression of SNAP-25 ${ }_{1-197}$ or BAPTA/AM treatment, both inhibiting calcium dynamics, facilitate block of GABAergic vesicle exocytosis upon toxin treatment. These data indicate that intrinsic calcium dynamics control botulinum A susceptibility in distinct neuronal populations.

\section{INTRODUCTION}

SNAP-25 belongs to the SNARE superfamily of membrane proteins that participate in synaptic vesicle (SV) exocytosis. It contributes two alpha-helices in the formation of the exocytotic fusion complex together with syntaxin-1 and synaptobrevin/VAMP [1, 2]. Besides acting as a fundamental component of the SNARE complex, SNAP-25 has been shown to negatively modulate voltage gated calcium channels in various overexpression systems $[3,4]$ and in cultured hippocampal neurons $[5,6]$.

Botulinum neurotoxin A (BoNT/A) is a protease specific for SNAP-25. Although the BoNT/A-generated fragment, SNAP-25 $5_{1-197}$, retains the capabillity to form the exocytotic fusion complex [7, 8], SNAP-25 proteolysis by the toxin reduces the probability of vesicle fusion and inhibits neurotransmitter release. It has been previously shown that BoNT/A preferentially inhibits SV recycling at glutamatergic terminals and is more efficient in impairing the release of excitatory than inhibitory neurotransmitters $[5,9,10,11]$. Lower susceptibility of GABAergic terminals to BoNT/A does not result from a defective toxin penetration, in line with the presence of the BoNT/A receptor SV2 in inhibitory neurons [11]. Notably the different susceptibility to BoNT/A of glutamatergic and GABAergic cells correlates with the levels of expression of the toxin substrate SNAP-25 in the two neuron types. Indeed, although most cultured GABAergic neurons use SNAP-25 for evoked exocytosis [12, 13, 14], they are characterized by lower levels of SNAP-25 as compared to glutamatergic terminals $[5,15,16,17]$ and become sensitive to BoNT/A upon exogenous expression of the protein [11]. Why BoNT/A is highly efficient in inhibiting vesicle cycling selectively in neurons expressing high levels of SNAP-25 is still to be defined. The clarification of 
this point may have important implications, especially in consideration of the wide use of BoNT/A for the treatment of human diseases, and in view of its employment in CNS pathologies [18, 19, 20].

\section{MATERIALS AND METHODS}

\subsection{Hippocampal neuronal cultures}

Primary cultures of rat hippocampal neurons were prepared from the hippocampi of 18 day-old fetuses as previously described [11]. Neurons were transfected using the calcium phosphate precipitation method. Silencing of SNAP-25 was achieved via transfection of a pSUPER construct provided by T. Galli (Paris, France) [5]. The cDNA

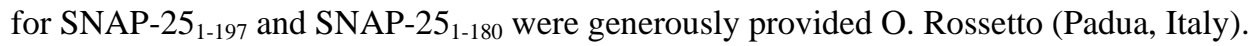

\subsection{Exo-endocytotic assay}

BoNT/A was a kind gift of Prof. Eric Johnson, Madison Wisconsin, purified and stored as previously described [11]. Control or transfected cultures were exposed to $125 \mathrm{nM}$ BoNT/A for 2 hours at $37^{\circ} \mathrm{C}$, before being assayed for SV recycling with monoclonal antibodies directed against the intravesicular domain of synaptotagmin I (Syt-ecto Abs; clone 604.2, kind gift of Dr. R. Jahn, Gottingen) as previously described [11]. Cultures were counterstained with polyclonal antibodies directed against vGLUT-1 (1:300, Synaptic Systems), vGAT (1:400-800; Synaptic Systems) or GAD (human sera recognizing GAD, kind gift of Dr. Solimena, Dresden). In a set of experiments recycling of GABAergic vesicles was evaluated using anti vGAT luminal antibodies (vGAT ecto, kind gift of Dr. H. Martens, Synaptic Systems). Cultures were co-stained with monoclonal anti-VGAT-N (Synaptic Systems). Images were acquired using Zeiss LSM 510 Meta confocal microscope and analyzed by Image J software. vGLUT positive recycling synapses, transfected with siRNA construct, were revealed by generating a binary mask of siRNA/vGLUT double-positive images. Total fluorescence (area per mean grey value) of Syt-ecto positive synapses expressing the siRNA construct, identified by the binary mask, were normalized to the total fluorescence of the corresponding v-GLUT staining. Syt-ecto/vGLUT fluorescence ratios of siRNA-transfected synapses were compared to those of non transfected vGLUT synapses. vGAT positive recycling synapses, transfected with SNAP25-HA fragments, were revealed by generating a binary mask of SNAP-25-HA/vGAT double-positive images. The number of Syt-ecto positive synapses expressing SNAP-25-HA, identified by the binary mask, was calculated. Synapses were scored as positive for Syt-ecto Ab internalization when the fluorescence intensity was at least 2.5 to 3 
times higher compared with cultures exposed only to secondary antibodies. For BAPTA experiments, vGAT positive synapses were revealed by generating a binary mask of vGAT images. Total fluorescence (area per mean grey value) of GABAergic Syt-ecto synapses, identified by the binary mask, were normalized to the total fluorescence of v-GAT staining. At least 10 fields containing excitatory/inhibitory synapses were analyzed, in three independent experiments. Quantitative analysis of SNAP-25 downregulation and overexpression in hippocampal cultures transfected with SNAP-25/GFP or cotransfected with iSNAP25 - GFP was carried out with monoclonal antibodies directed against SNAP-25 (SMI81, Sternberger Monoclonals, Baltimore MD). Images were acquired using Zeiss LSM 510 Meta confocal microscope and analyzed by Image J-1.4.3.67 NIH software. SNAP-25 expression was measured as mean grey value in neuronal processes of transfected and non-transfected cells.

\subsection{Calcium measurements}

Cultures were loaded with the ratiometric calcium dye FURA-2/AM as previously described [5].

Polychrome IV (TILL Photonics, Grafelfing, Germany) was used as a light source. Fura-2 fluorescence images were collected with a PCO Super VGA SensiCam (Axon Instruments, Forest City, CA, USA) and analysed with TILLvision software. After excitation at 340 and $380 \mathrm{~nm}$ wavelengths, the emitted light was acquired at $505 \mathrm{~nm}$ at 1-4 Hz. The ratio values in discrete areas of interest were calculated from sequences of images to obtain temporal analyses. Calcium concentrations were expressed as F340/380 fluorescence ratios.

\subsection{Statistical analysis}

Results are presented as means \pm S.E. Data were statistically compared using the Student’s t test by using Origin software (Origin Lab, Northampton). One asterisk means $\mathrm{p}<0.05$, two asterisks $\mathrm{p}<0.01$.

\section{RESULTS}

\subsection{Levels of SNAP-25 expression influence neuron sensitivity to BoNT/A}

We have previously shown that GABAergic SV exocytosis, monitored by antibodies to the intravesicular domain of synaptotagmin I (Syt-ecto), is highly resistant to BoNT/A [5, 11]. By monitoring GABAergic vesicle fusion with antibodies recognizing the C-terminus of the vesicular GABA transporter vGAT (vGAT ecto) [21], we confirmed that the majority of GABAergic terminals, intoxicated with $125 \mathrm{nM}$ BoNT/A, internalized vGAT ecto 
antibodies upon exposure to $55 \mathrm{mM} \mathrm{KCl}$ for $4 \mathrm{~min}$; exocytosis was instead efficiently blocked by tetanus toxin (20 nM, TeNT) which cleaves synaptobrevin/VAMP-2 (figure 1A). Conversely, BoNT/A treatment inhibited SV recycling at excitatory synapses, monitored by antibodies to the intravesicular domain of synaptotagmin I (Syt-ecto) (figure 1B). SNAP-25 overexpression in GABAergic terminals was reported to largely increase sensitivity to BoNT/A and prevent Syt-ecto internalization [11]. To complement this finding and confirm that the expression level of SNAP-25 is an important determinant in neuronal sensitivity to BoNT/A, we investigated the effect of reducing SNAP-25 expression in glutamatergic neurons by co-transfection of cDNA codifying nucleotides 321 to 339 of rat SNAP-25 together with GFP (siRNA sequence). SNAP-25 expression was reduced of about $60 \%$ at 1-2 days after transfection (figure 1C, quantitation) and progressively decreased to levels not reliably quantifiable (figure 1C, arrow). Downregulation of SNAP-25 did not impair Syt-ecto Abs internalization at excitatory terminals (figure 1D), thus implying that low levels of the protein are sufficient to mediate SV fusion, as already demonstrated in cultures derived from SNAP-25 heterozygous mice [13, F. Antonucci, I. Corradini, C. Verderio and M.Matteoli, unpublished observations]. Cultures co-transfected with SNAP-25 siRNA sequence and GFP were exposed to BoNT/A and SV recycling was assayed using Syt-ecto Abs. A larger resistance to BoNT/A was detected at SNAP-25-silenced excitatory synapses. Indeed, the percentage of excitatory synapses resistant to intoxication raised from about $20 \%$ in controls to $40 \%$ after siRNA treatment (figure $1 \mathrm{E}$ ). Furthermore the extent of recycling in SNAP-25-silenced boutons was higher as compared to the BoNT/A resistant terminals, either non transfected or GFP transfected (figure $1 \mathrm{E}$ and F). Overall these data support the concept that variations in the expression of the SNARE at nerve terminals underlie differences in BoNT/A sensitivity.

\subsection{Reduction of neuronal calcium transients enhances sensitivity of GABAergic terminals to BoNT/A}

BoNT/A cleaves SNAP-25 at residue 197 to generate a membrane bound $\mathrm{NH}_{2}$-terminal SNAP-251-197 fragment, while BoNT/E removes a larger C terminus region of the protein by cleaving SNAP-25 at residue 180 . The 17 amino acids between the cleavage sites for BoNT/A and BoNT/E are known to be required for high affinity binding of SNAP-25 to syntaxin 1 [22], for ternary complex formation with VAMP-2 and syntaxin 1 [7], for $\mathrm{Ca}^{2+}$ triggered membrane fusion [23] and $\mathrm{Ca}^{2+}$-dependent synaptotagmin binding [24]. In addition, the C-terminal region of SNAP-25 between BoNT/A and BoNT/E cleavage sites is also required for SNAP-25 modulation of voltage gated calcium currents [5, 6].

To get insights into the mechanisms by which overexpression of SNAP-25 confers GABAergic neurons with BoNT/A sensitivity [11], hippocampal cultures were transfected with plasmids encoding portions of SNAP-25 
(SNAP-25 ${ }_{1-180}$ and SNAP-25 ${ }_{1-197}$ ) fused to a HA-tag. SV fusion was monitored in transfected GABAergic terminals upon BoNT/A intoxication. While no significant reduction of SV exocytosis was detected at intoxicated GAD positive synapses expressing the SNAP-25 $5_{1-180}$ fragment, SV fusion was strongly impaired at GAD positive synapses expressing SNAP-25 1 -197 (figure 2A-C). SV recycling was only analyzed at terminals where the expression levels of the transfected fragment was increased of about 2 folds, excluding those terminals expressing higher protein amounts (3-8 folds, figure 2C). This effect was not due to an inhibitory action of SNAP-25 ${ }_{1-197}$ fragment on SV fusion, since exogenous expression of the fragment in inhibitory neurons did not impair vesicle recycling (figure 2D). These results indicate that SNAP-25 ${ }_{1-197}$, but not SNAP-25 $5_{1-180}$ is able to enhance GABAergic neuron sensitivity to BoNT/A, similarly to full length SNAP-25 [11] .

It has been previously shown that BoNT/A-inhibition of SV fusion can be overcome by high calcium concentrations [25, 26, 27]. Based on this finding, Delgado-Martinez et al. [12] suggested that the lower efficacy of BoNT/A in inhibiting GABAergic vesicle cycling might be attributable to the higher calcium responsiveness of GABAergic relative to glutamatergic neurons. Given SNAP-25 ${ }_{1-197}$ but not SNAP-25 $1-180$ negatively modulates evoked calcium responses [5], the possibility opens that SNAP-25 1 -197, as the full length protein, enhances BoNT/A sensitivity by reducing calcium currents. To directly address this hypothesis, cultures were treated with the membrane permeable calcium chelator BAPTA/AM $(25 \mu \mathrm{M})$ for 15 or 20 min. BAPTA/AM incubation for 15 min reduced by about 30\% peak calcium transients evoked by $55 \mathrm{mM} \mathrm{KCl}$ (figure 3A, B) and only mildly affected depolarization-induced SV fusion at GABAergic synapses (fig. 3C-D). Of note, BAPTA/AM largely increased the sensitivity of GABAergic terminals to BoNT/A (figure $3 \mathrm{E}$ and F). Although monitoring of $\mathrm{Ca}^{2+}$ elevation was carried out in neuronal cell bodies, these data suggest that the higher $\left[\mathrm{Ca}^{2+}\right]_{\mathrm{i}}$ responses at GABAergic terminals can overcome the blocking action of the toxin.

\section{DISCUSSION}

Given that BoNTs do not cross the blood-brain barrier, their action is normally limited to peripheral nerve terminals. However, when injected directly into the brain, BoNTs can intoxicate neurons of the central nervous tissue, which express both substrates and receptors of BoNTs, with specific patterns of distribution. The preferential expression of receptors/substrates at specific neurons might underlie the preferential efficiency of BoNTs at selected terminals [20]. In the case of BoNT/A, however, the different sensitivity of glutamatergic and GABAergic neurons to the toxin does not result from receptor expression [11]. It does not result either from the presence in GABAergic neurons of a BoNT/A-resistant substrate, such as SNAP-47 or SNAP-29, since SNAP-25 has been shown to 
mediate GABA release in primary cultures $[12,13,14]$. Here we show that two treatments which reduce evoked calcium transients, ie transfection of SNAP-25 ${ }_{1-197}$ and exposure to BAPTA-AM, increase susceptibility of GABaergic neurons to BoNT/A. Conversely, downregulation of SNAP-25, which increases evoked calcium transients [5], makes glutamatergic neurons more resistant to BoNT/A. These data suggest that susceptibility of neurons to BoNT/A results from the intrinsic calcium responsiveness to depolarization, which, among different factors, is modulated by the level of expression of SNAP-25 [5, 6, 28]. Interestingly, different intracellular calcium dynamics do not affect toxin penetration [11]. Our data are consistent with previous evidences obtained in hippocampal slice cultures, where significant increases in mEPSC frequency were produced in BoNT/A-treated cultures by application of the $\mathrm{Ca}^{2+}$ ionophore ionomycin in the presence of $10 \mathrm{mM}$ [Ca2+] [25]. Similarly, elevating calcium concentration at the calyx of Held with the use of caged calcium, produced a strong reduction in the calcium sensitivity for release after the cleavage of SNAP-25 by BoNT/A [27]. Interestingly, besides the amplitude of the peak calcium transients, also the temporal pattern of depolarization-induced calcium elevations influences neuronal BoNT/A susceptibility. Indeed, BoNT/A has been reported to abolish CGRP release induced by high $\mathrm{KCl}$ or bradykinin in trigeminal ganglionic neurons, but not that induced by capsaicin, which evokes a more prolonged calcium response as compared to the other stimuli [8]. Under this respect, it is of note that GABAergic neurons display not only higher but also slowly inactivating calcium currents compared to glutamatergic neurons (S. Condliffe, I. Corradini , D. Pozzi, C.Verderio and M. Matteoli, unpublished results). Altogether these data indicate that, in addition to receptor and/or substrate expression, a different susceptibility to BoNT/A may also result from intrinsic calcium dynamics in distinct neuronal populations. These data add a relevant information for the use of BoNT/A in the therapy of neurological disorders caused by the unbalanced activity of excitatory neuronal populations where maintenance of GABAergic transmission could be advantageous. Among these disorders are epilepsy [19] and neuropathic pain [29].

\section{ACKNOWLEDGMENTS}

We thank Fondazione Italo Monzino for donating Zeiss LSM 510 Meta. This work was supported by EUSynapse Integrated Project, Cariplo (2006.0779/10.9251) and Compagnia San Paolo (Prog. 2005.1964).

\section{REFERENCES}

[1] R. Jahn, R.H. Scheller, SNAREs--engines for membrane fusion, Nat Rev Mol Cel. 7 (2006) 631-643.

[2] T.C. Südhof, J.E. Rothman, Membrane fusion: grappling with SNARE and SM proteins, Science. 323 (2009) 474477. 
[3] O. Wiser, M. Trus, A. Hernández, et al., The voltage sensitive Lc-type Ca2+ channel is functionally coupled to the exocytotic machinery, Proc Natl Acad Sci U S A. 96 (1999) 248-253.

[4] H. Zhong, C.T. Yokoyama, T. Scheuer, W.A. Catterall, Reciprocal regulation of P/Q-type Ca2+ channels by SNAP25, syntaxin and synaptotagmin, Nat Neurosci. 2 (1999) 939-941.

[5] C. Verderio, D. Pozzi, E. Pravettoni, et al., SNAP-25 modulation of calcium dynamics underlies differences in GABAergic and glutamatergic responsiveness to depolarization, Neuron. 41 (2004) 599-610.

[6] D. Pozzi, S. Condliffe, Y. Bozzi, et al., Activity-dependent phosphorylation of Ser187 is required for SNAP-25negative modulation of neuronal voltage-gated calcium channels, Proc Natl Acad Sci U S A. 105 (2008) 323-328.

[7] T. Hayashi, H. McMahon, S. Yamasaki, et al., Synaptic vesicle membrane fusion complex: action of clostridial neurotoxins on assembly, EMBO J. 13 (1994) 5051-5061.

[8] J. Meng, S.V. Ovsepian, J. Wan, et al., Activation of TRPV1 mediates calcitonin gene-related peptide release, which excites trigeminal sensory neurons and is attenuated by a retargeted botulinum toxin with anti-nociceptive potential, $\mathrm{J}$ Neurosci. 29 (2009) 4981-4992.

[9] H. Bigalke, I. Heller, B. Bizzini, E. Habermann. Tetanus toxin and botulinum A toxin inhibit release and uptake of various transmitters, as studied with particulate preparations from rat brain and spinal cord, Naunyn Schmiedebergs Arch Pharmaco. 316 (1981) 244-251.

[10] A. Ashton , J.O. Dolly, Characterization of the inhibitory action of botulinum neurotoxin type A on the release of several transmitters from rat cerebrocortical synaptosomes, J Neurochem. 50 (1988) 1808-1816.

[11] C. Verderio, C. Grumelli, L. Raiteri, Coco S, et al., Traffic of botulinum toxins A and E in excitatory and inhibitory neurons, Traffic. 8 (2007) 142-153.

[12] I. Delgado-Martínez, R.B. Nehring, J.B. Sørensen, Differential abilities of SNAP-25 homologs to support neuronal function, J Neurosci. 27 (2007) 9380-9391.

[13] P. Bronk, F. Deák, M.C. Wilson, X. Liu, T.C. Südhof, E.T. Kavalali, Differential effects of SNAP-25 deletion on Ca2+ -dependent and Ca2+ -independent neurotransmission, J Neurophysiol 98 (2007) 794-806.

[14] L.C. Tafoya, M. Mameli, T. Miyashita, J.F. Guzowski, C.F. Valenzuela, M.C. Wilson, Expression and function of SNAP-25 as a universal SNARE component in GABAergic neurons, J Neurosci. 26 (2006) 7826-7838.

[15] L. Bragina, C. Candiracci, P. Barbaresi, S. Giovedì, F. Benfenati, F. Conti, Heterogeneity of glutamatergic and GABAergic release machinery in cerebral cortex, Neuroscience 146 (2007) 1829-1840. 
[16] R. Garbelli, F. Inverardi, V. Medici, et al., Heterogeneous expression of SNAP-25 in rat and human brain, J Comp Neurol. 506 (2008) 373-386.

[17] G. Mandolesi, V. Vanni, R. Cesa, et al., Distribution of the SNAP25 and SNAP23 synaptosomal-associated protein isoforms in rat cerebellar cortex, Neuroscience. 164 (2009) 1084-1096.

[18] G. Schiavo, M. Matteoli, C. Montecucco, Neurotoxins affecting neuroexocytosis, Physiol Rev. 80 (2000) 717-766.

[19] L. Costantin, Y. Bozzi, C. Richichi, et al., Antiepileptic effects of botulinum neurotoxin, E J Neurosci, 25 (2005) 1943-1951.

[20] C. Verderio, O. Rossetto, C. Grumelli, C. Frassoni, C. Montecucco, M. Matteoli, Entering neurons: botulinum toxins and synaptic vesicle recycling, EMBO Rep. 7 (2006) 995-999.

[21] H. Martens, M.C. Weston, J.L. Boulland, et al., Unique luminal localization of VGAT-C terminus allows for selective labeling of active cortical GABAergic synapses, J Neurosci. 28 (2008) 13125-13131.

[22] M. Bajohrs, C. Rickman, T. Binz, B. Davletov, A molecular basis underlying differences in the toxicity of botulinum serotypes A and E, EMBO Rep. 5 (2004) 1090-1095.

[23] A. Banerjee, J.A. Kowalchyk, B.R. DasGupta, T.F. Martin, SNAP-25 is required for a late postdocking step in Ca2+ dependent exocytosis, J Biol Chem. 271 (1996) 20227-20230.

[24] R.R. Gerona, E.C. Larsen, J.A. Kowalchyk, T.F. Martin, The C terminus of SNAP25 is essential for Ca(2+)dependent binding of synaptotagmin to SNARE complexes, J Biol Chem. 275 (2000) 6328-6336.

[25] M. Capogna, R.A. McKinney, V. O'Connor, B.H. Gähwiler, S.M. Thompson, Ca2+ or Sr2+ partially rescues synaptic transmission in hippocampal cultures treated with botulinum toxin A and C, but not tetanus toxin, J Neurosci. 17 (1997) 7190-7202.

[26] L.E. Trudeau, Y. Fang, P.G. Haydon, Modulation of an early step in the secretory machinery in hippocampal nerve terminals, Proc Natl Acad Sci U SA. 95 (1998) 7163-7168.

[27] T. Sakaba, A. Stein, R. Jahn, E. Neher, Distinct kinetic changes in neurotransmitter release after SNARE protein cleavage, Science. 309 (2005) 491-494.

[28] C. Grumelli, P. Berghuis, D. Pozzi, Caleo et al., Calpain activity contributes to the control of SNAP-25 levels in neurons, Mol Cell Neurosci. 39 (2008) 314-332. 
[29] Y. Kitamura, Y. Matsuka, I. Spigelman, et al., Botulinum toxin type a (150 kDa) decreases exaggerated neurotransmitter release from trigeminal ganglion neurons and relieves neuropathy behaviors induced by infraorbital nerve constriction, Neuroscience. 159 (2009) 1422-1429.

\section{FIGURE LEGENDS}

Figure 1 Downregulation of SNAP-25 at glutamatergic synapses reduces BoNT/A sensitivity

A, B Uptake of vGAT ecto (A, green) or Syt ecto Abs (B, red) at inhibitory terminals stimulated with $55 \mathrm{mM} \mathrm{KCl}$ in control neurons or in neurons intoxicated with BoNT/A or TeNT. Internalized antibodies were visualized after fixation and permeabilization by indirect immunofluorescence. Neurons were counterstained for vGAT-N (A, red) or vGLUT-1 (B, green). C, SNAP-25 immunoreactivity (red) at vGLUT-1 positive glutamatergic synapses (blue), co-transfected with SNAP-25 siRNA construct and GFP (green), 4-5 days after transfection. Arrow indicates a transfected glutamatergic synapse, virtually devoid of SNAP-25. The histogram shows mean gray value detected in neuronal processes of non transfected and siRNA transfected neurons, 1-2 days after transfection. D, Cultures transfected with SNAP-25 siRNA construct and GFP (green) were incubated with Syt-ecto Ab (red) under depolarizing conditions and counterstained with antibodies anti-vGLUT-1 (blue). E, In BoNT/A-intoxicated cultures the Syt-ecto Abs are more efficiently internalized at SNAP-25 siRNA and GFP co-transfected synapses than at control synapses. F, Histogram shows the quantitative analysis of SV recycling at siRNA GFP co-transfected (SNAP-25 siRNA), non transfected (control) or GFP transfected (GFP) glutamatergic synapses .Scale bar: $4 \mu \mathrm{m}$ in A, B and $\mathrm{E} ; 5 \mu \mathrm{m}$ in $\mathrm{C}$ and $\mathrm{D}$.

Figure 2 Expression of SNAP-25 ${ }_{\text {1-197 }}$ but not SNAP-25 ${ }_{\text {1-180 }}$ enhances sensitivity of GABAergic synapses to BoNT/A

A and B, Cultures transfected with SNAP-25 1-197 or SNAP-25 1-180 fused to a HA tag (red) were intoxicated with BoNT/A, incubated with Syt-ecto Abs (blue) and counterstained with antibodies against GAD (green). While SNAP-25 1-180 expression does not significantly alter SV recycling, SNAP-25 1-197 confers GABAergic synapses with BoNT/A sensitivity. C, Quantitative analysis of SNAP-25 1-197 overexpression (mean gray value) in transfected processes as compared to non transfected ones. Neurons characterized by an expression ratios exceeding three-fold were discarded from the analysis of SV recycling shown in D. D, Quantitative analysis of SV recycling at SNAP$25_{1-197}$ or SNAP-25 1-180 transfected GABAergic synapses as compared to non transfected ones. E, Cultures transfected with SNAP-25 1-197 fused to a HA tag (green) were incubated with Syt-ecto Abs (red) under depolarizing 
conditions and counterstained with antibodies against GAD (blue). Note that Syt-ecto Abs are efficiently internalized in GABAergic processes expressing SNAP-25 1- 197 (see also inset). Number of analyzed interneurons expressing SNAP-25 1-197 =33; number of GFP transfected interneurons =40; number of experiments: 5 .Scale bar: 8 $\mu \mathrm{m}$ in $\mathrm{A}, 7 \mu \mathrm{m}$ inset; $8 \mu \mathrm{m}$ in $\mathrm{B} ; 9 \mu \mathrm{min} \mathrm{D}, 4 \mu \mathrm{m}$ inset.

\section{Figure 3 BAPTA-AM treatment enhances sensitivity of GABAergic synapses to BoNT/A}

A, Temporal analysis of calcium transients induced in FURA-2 loaded neurons by $55 \mathrm{mM} \mathrm{KCl}$ before and after incubation with BAPTA-AM. Calcium concentrations are indicated as 340/380 fluorescence ratios. B, Quantitative analysis of calcium transient amplitude after BAPTA-AM treatment for 15 or 20 min. C, D, Uptake of Syt ecto Abs (red) at vGAT positive inhibitory terminals (green) in control or BAPTA-AM- treated cultures (15 min).

Quantitative analysis of Syt ecto Abs internalization is shown in D. E and F, Uptake of Syt ecto Abs (red) at vGAT positive synapses (green) in BoNT/A intoxicated cultures incubated or not with BAPTA-AM. Note that GABAergic vesicle fusion is more efficiently inhibited by BoNT/A in cultures exposed to BAPTA-AM (F) Scale bar: $10 \mu \mathrm{m}$ in $\mathrm{C}, 8 \mu \mathrm{m}$ in $\mathrm{E}$. 


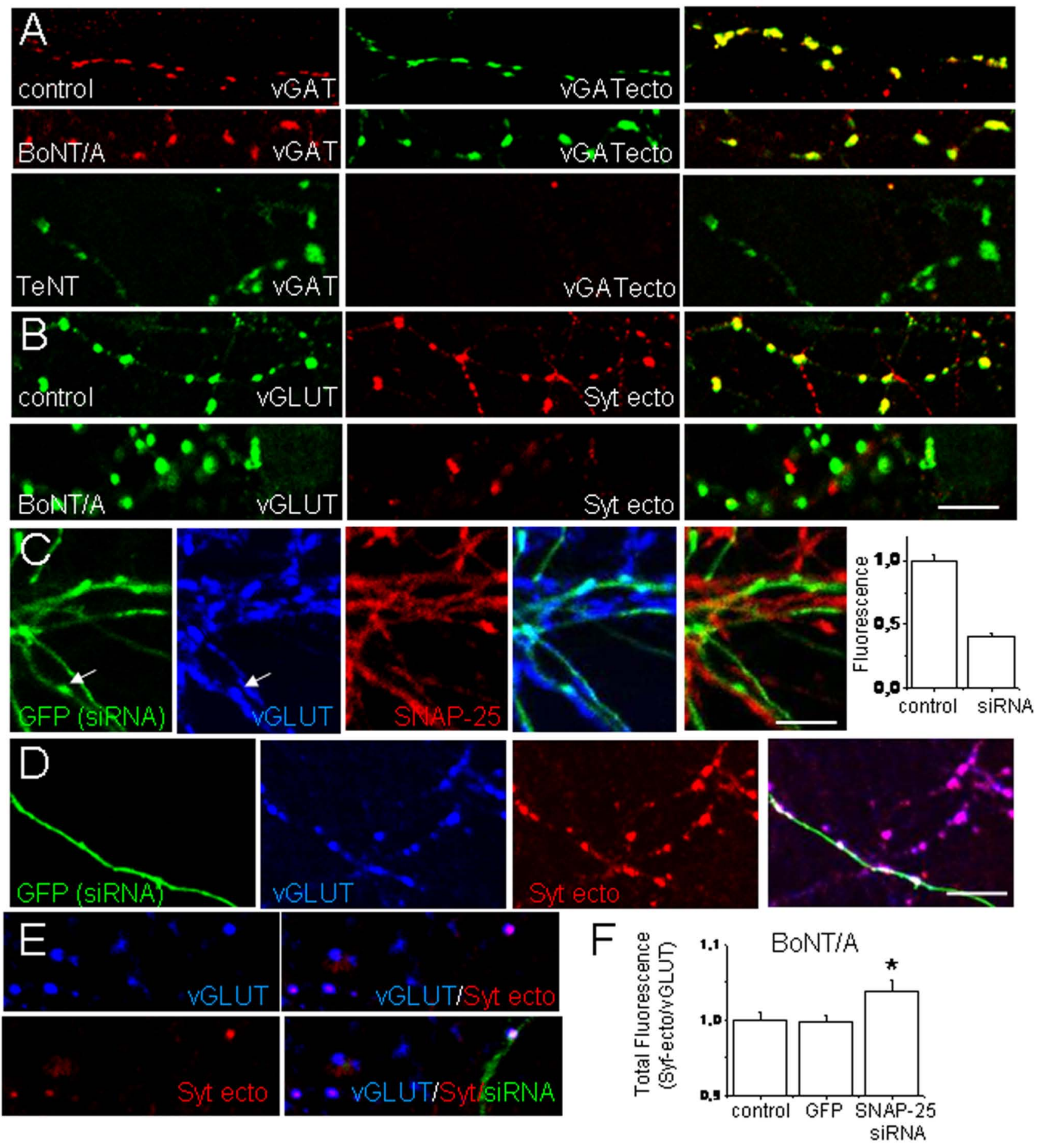


Figure 2
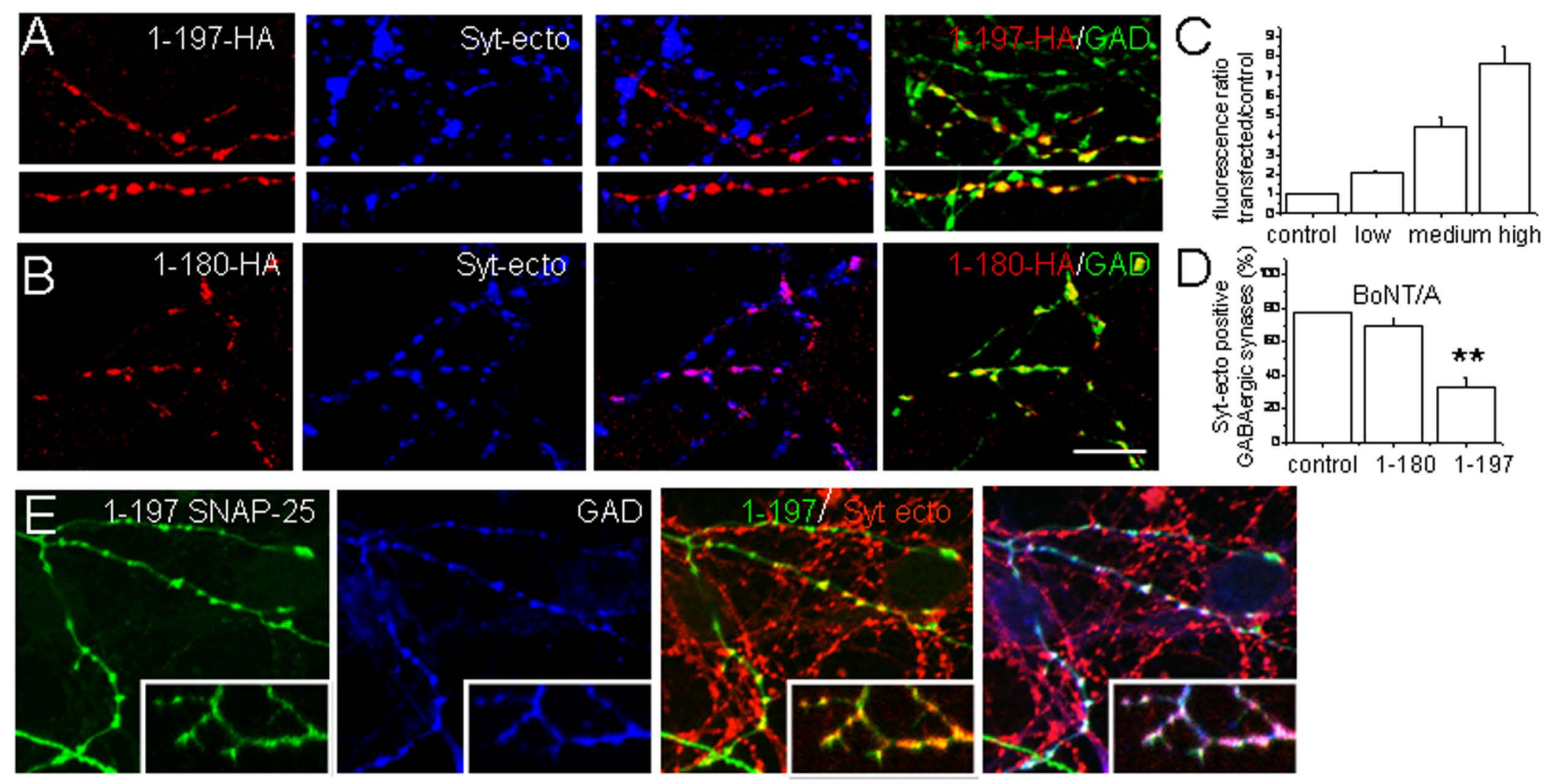
Figure 3
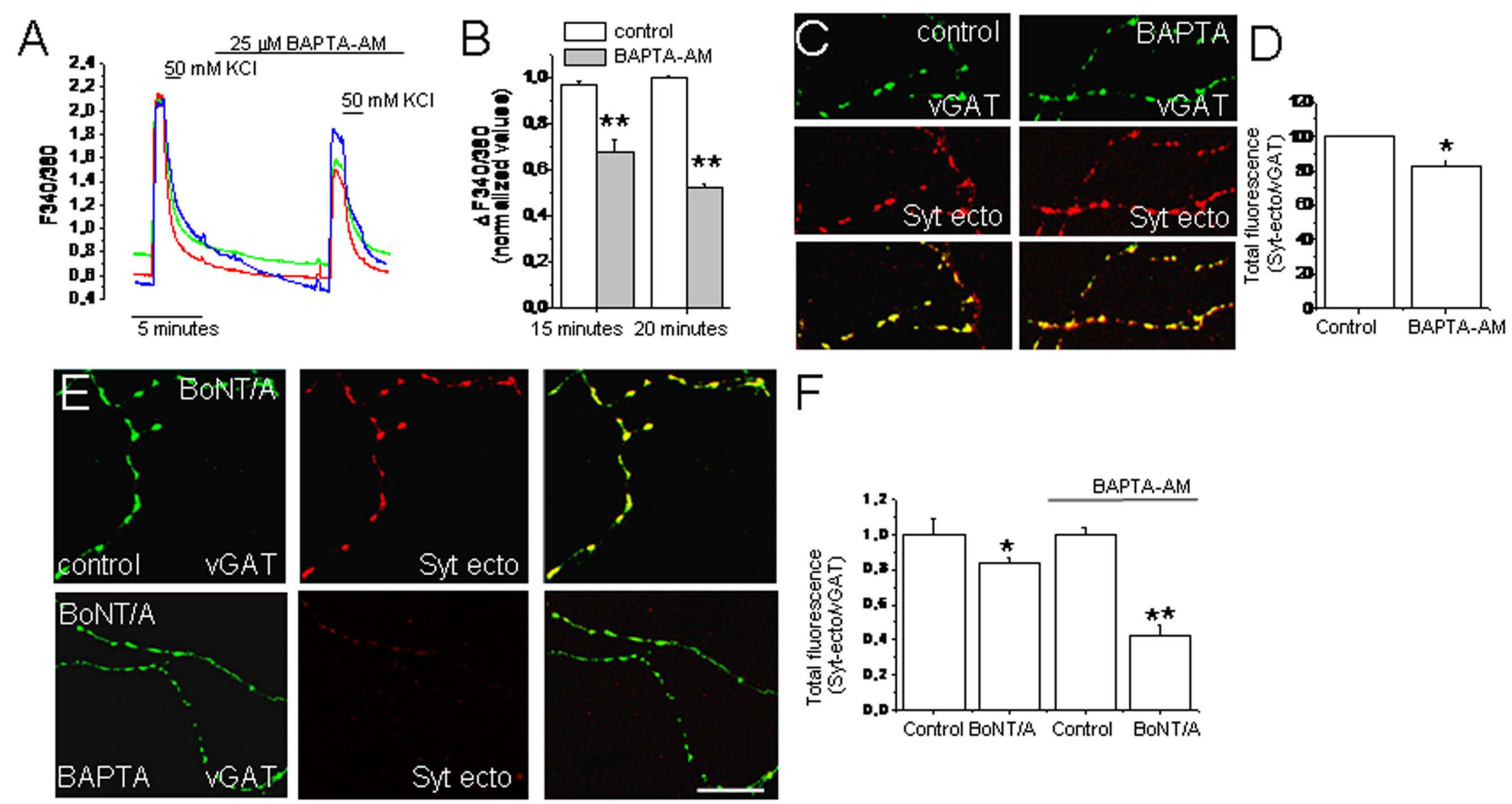